\title{
Effects of selenium on myocardial apoptosis by modifying the activity of mitochondrial STAT3 and regulating potassium channel expression
}

\author{
CHANGJIANG ZHANG ${ }^{1}$, YINZHI DENG $^{2}$, YUHUA LEI $^{1}$, JINGBO ZHAO $^{1}$, WEN WEI $^{1}$ and YUANHONG LI $^{1}$ \\ ${ }^{1}$ Cardiovascular Disease Center and ${ }^{2}$ Department of Gastroenterology, Central Hospital \\ of Enshi Autonomous Prefecture, Enshi, Hubei 445000, P.R. China
}

Received January 25, 2017; Accepted June 19, 2017

DOI: $10.3892 / \mathrm{etm} .2017 .4716$

\begin{abstract}
The present study investigated the effects of myocardial mitochondrial signal transduction and activator of transcription 3 (STAT3), succinate dehydrogenase activity and changes of potassium channel expression on cardiomyocyte apoptosis under low selenium conditions. Primary cultured cardiomyocytes from neonatal mice were divided into the non-toxic control group ( $0.1 \mu \mathrm{M}$ sodium selenite $)$ and low selenium treatment group $(0.05 \mu \mathrm{M}$ sodium selenite $)$ according to different selenium concentrations. The expression of mitochondrial STAT3, p-STAT3, p-Kv1.2 potassium channel and apoptosis-related proteins, Bax and Bcl-2, were assessed by immunoblotting. Succinate dehydrogenase activity was measured by spectrophotometry. Flow cytometry was used to detect cardiomyocyte apoptosis. Low selenium treatment reduced the expression of p-STAT3, but did not affect the expression of STAT3. In addition, low selenium treatment reduced the activity of mitochondrial STAT3 and succinate dehydrogenase in cardiomyocytes, leading to injury of myocardial mitochondria. Compared with the control group, low selenium conditions reduced the activity of p-Kv1.2 and reduced the normal electrophysiological function of cardiomyocytes. In the low selenium-treated group, the expression of Bax protein increased, whereas the expression of Bcl-2 protein decreased. The apoptotic rate increased. In conclusion, selenium deficiency in cardiomyocytes leads to decreased potassium channel expression and decreased mitochondrial STAT3 activity and mitochondrial function, which in turn promotes the apoptosis of cardiomyocytes.
\end{abstract}

Correspondence to: Professor Yuanhong Li, Cardiovascular Disease Center, Central Hospital of Enshi Autonomous Prefecture, 158 Wuyang Street, Enshi, Hubei 445000, P.R. China

E-mail:vmw3y6@163.com

Key words: low selenium, STAT3, potassium channel protein, apoptosis

\section{Introduction}

As an essential trace element for humans, selenium plays pivotal regulatory functions in maintaining human health by supporting the function of various enzymes (1). Previous experimental and clinical studies have shown that selenium level and cardiovascular disease are correlated. The incidence of coronary heart disease is negatively correlated with blood selenium levels, and blood selenium level is an important indicator for the determination of coronary heart disease (2). Atherosclerosis is primarily caused by the peroxidation of endothelial cell membrane lipids. Low selenium leads to decreased activity of antioxidant enzymes, resulting in the aggravation of atherosclerosis. Selenium supplements have significant therapeutic effects in patients with atherosclerosis (3). In addition, low selenium is a major cause of Keshan disease, which manifests with myocardial necrosis (4). Therefore, selenium plays an important role in the apoptosis of cardiomyocytes. STAT3 is important in signal transduction and the formation of various cardiac cells. STAT3 has a variety of functions, which include effects on mitochondrial transport pores and the regulation of energy metabolism. Changes in mitochondrial STAT3 expression and activity can reflect myocardial cell activity (5). Succinate dehydrogenase is located in the mitochondrial inner membrane, and is involved in mitochondrial ATP production. Changes in the levels of succinate dehydrogenase can reflect intracellular mitochondrial activity. Myocardial repolarization current abnormalities and the prolongation of action potential duration are important causes for the development of arrhythmia, in which potassium channel-mediated $\mathrm{K}^{+}$currents play an important role. In addition, heart failure affects the expression of various potassium channels (6). Potassium channels and apoptosis are closely related. The loss of potassium ions can promote the apoptosis of normal cells and further lead to the occurrence and aggravation of myocardial disease (7).

Low selenium affects the expression of mitochondrial STAT3 and potassium channels (8), although its relationship with cardiomyocyte apoptosis remains unclear. To investigate the effects of low selenium on mitochondrial activity and myocardial cell apoptosis and the molecular mechanism, the levels of STAT3, succinate dehydrogenase, the Kv1.2 potassium channel, and apoptosis-related proteins in primary 
cultured cardiomyocytes of neonatal mice were measured under low selenium and normal selenium conditions.

\section{Materials and methods}

Extraction and culture of neonatal rat cardiomyocytes. Six 3-day-old C57BL/6 mice from the Model Animal Research Center of Nanjing (Nanjing, China), were immersed in $75 \%$ absolute ethanol for 1-2 min. The sternum was cut to harvest the heart. The heart was then placed in precooled Hanks broth to remove excess surrounding tissue, and rinsed three times with precooled Hanks broth. The heart was then placed in trypsin and sectioned. Samples were placed in a $37^{\circ} \mathrm{C}$ water bath for $10 \mathrm{~min}$. The supernatant was removed after precipitation, followed by additional trypsin digestion for $10 \mathrm{~min}$. The samples were mixed with a pipette and medium was added to stop the digestion. The digestion process was repeated 6 times. The digested cells were centrifuged at $111.8 \mathrm{x} \mathrm{g}$ for $10 \mathrm{~min}$, and mixed with an appropriate volume of medium. Finally, the samples were cultured in an incubator with $5 \% \mathrm{CO}_{2}$ at $37^{\circ} \mathrm{C}$. Cell status was observed $24 \mathrm{~h}$ later.

Approval for animal studies was obtained from the Ethics Committee of Central Hospital of Enshi Autonomous Prefecture.

Determination of the concentration of low selenium culture medium. Well-grown primary cardiomyocytes were cultured in medium containing $0,0.01,0.05,0.08,0.1,0.5$ and $1 \mu \mathrm{M}$ sodium selenite, and normal culture medium, respectively. After $36 \mathrm{~h}$, the cell death rates under different concentrations of selenium were calculated. Lactate dehydrogenase test showed no significant difference in toxicity between the $0.1 \mu \mathrm{M}$ selenium treatment and normal culture medium groups. The $0.1 \mu \mathrm{M}$ selenium treatment group was suitable for use as the normal selenium control group. Compared with the $0.1 \mu \mathrm{M}$ selenium treatment, medium containing higher or lower selenium concentrations resulted in different degrees of cell death. Cardiomyocyte mortality was $50 \%$ in the group treated with $0.05 \mu \mathrm{M}$ selenium. Medium containing $0.05 \mu \mathrm{M}$ selenium was used in the low selenium-treated group.

Extraction of myocardial mitochondria. Appropriate amounts of cardiomyocytes were collected from the control and low selenium treatment groups. The cells were digested with trypsin. After centrifugation, appropriate amounts of lysis buffer were added, and the samples were placed in a homogenizer and ground on ice. Nuclei were removed by centrifugation at $1,200 \mathrm{x} \mathrm{g}$ for $15 \mathrm{~min}$ at $4^{\circ} \mathrm{C}$, followed by centrifugation at $10,000 \mathrm{x}$ g for $10 \mathrm{~min}$. The supernatant was discarded, and the mitochondrial precipitate was retained. Finally, an appropriate amount of mitochondrial storage solution was added for $-80^{\circ} \mathrm{C}$ storage of samples.

Detection of mitochondrial succinate dehydrogenase activity. A succinate dehydrogenase activity assay kit (Sigma-Aldrich, St. Louis, MO, USA) was used. Samples and standards for analysis were diluted according to the instructions of the kit, and were added to enzyme-linked immunosorbent assay plates. Plates were blocked and placed at $37^{\circ} \mathrm{C}$ for $30 \mathrm{~min}$. The liquid was discarded, samples were washed with washing buffer, and
$50 \mu 1$ of enzyme-labeled reagent was added to each well. The plates were then subjected to $37^{\circ} \mathrm{C}$ incubation for $30 \mathrm{~min}$. The color development step was performed in the dark at $37^{\circ} \mathrm{C}$ for $10 \mathrm{~min}$ after five washes. Succinate dehydrogenase activity was measured at $600 \mathrm{~nm}$ using a spectrophotometer (Thermo Fisher Scientific, Waltham, MA, USA).

Immunoblotting. Mitochondria from myocardial cells in the control and low selenium treatment groups were harvested, and protein concentration was measured with a NanoDrop 2000 (Thermo Fisher Scientific) after lysis. In addition, whole cell protein concentration in the control and low selenium treatment groups were measured after whole cell lysis. Equal amounts of mitochondrial lysate or whole cell lysate were subjected to SDS-PAGE. After electrophoresis at $120 \mathrm{~V}$ for $80 \mathrm{~min}$, the protein was transferred to membranes under $350 \mathrm{~mA}$ for $4 \mathrm{~h}$. The membranes were blocked with 5\% sugar-free skim milk for $1 \mathrm{~h}$. After incubation with primary antibody for $4 \mathrm{~h}$ at room temperature according to the instructions, the membranes were washed three times with TBST and incubated with secondary antibody for $1 \mathrm{~h}$ at room temperature, followed by radiography. Rabbit monoclonal STAT3 antibody (dilution, 1:500; cat. no. ab68153), rabbit monoclonal p-STAT3 antibody (dilution, 1:500; cat. no. ab76315), rabbit polyclonal Kv1.2 antibody (dilution, 1:500; cat. no. ab55987), rabbit polyclonal p-Kv1.2 antibody (dilution, 1:500; cat. no. ab65789) and secondary goat anti-rabbit (HRP) IgG antibody (dilution, 1:2,000; cat. no. ab6721) were all purchased from Abcam (Cambridge, MA, USA). The images obtained were scanned. Image J (National Institutes of Health, Bethesda, MD, USA) was used to analyze grayscale values for each band and the data were recorded for statistical analysis. Antibodies used for immunoblotting were from Abcam (Chicago, IL, USA).

Flow cytometric detection of myocardial cell apoptosis. Cardiomyocytes were collected from the normal control and low selenium treatment groups. After washing with 0.01 MPBS, the cells were fixed in precooled $70 \%$ ethanol for $2 \mathrm{~h}$. The cells were then resuspended in PBS and filtered, followed by centrifugation at $111.8 \mathrm{x}$ g for $5 \mathrm{~min}$. PBS was discarded. The samples were incubated with propidium iodide (PI) staining solution for $0.5 \mathrm{~h}$ at $4^{\circ} \mathrm{C}$ in the dark. Cardiomyocyte apoptosis was measured using flow cytometry (BD Biosciences, San Jose, CA, USA) at $488 \mathrm{~nm}$.

Statistical analysis. Statistical analysis was performed using SPSS 17.0 (SPSS, Inc., Chicago, IL, USA). Data are presented as mean \pm standard deviation. Differences between groups were analyzed by t-test. $\mathrm{P}<0.05$ was considered statistically significant.

\section{Results}

Determination of myocardial mitochondrial enzyme activity and changes of STAT3 expression. After $36 \mathrm{~h}$ of culture, myocardial mitochondria of the control and low selenium-treated groups were extracted, and succinate dehydrogenase activity was measured. Compared with the control group, succinate dehydrogenase activity in the low selenium group was significantly decreased $(\mathrm{P}<0.05)($ Fig. 1A). p-STAT3 is the active state of STAT3. After mitochondrial lysis, the 
A

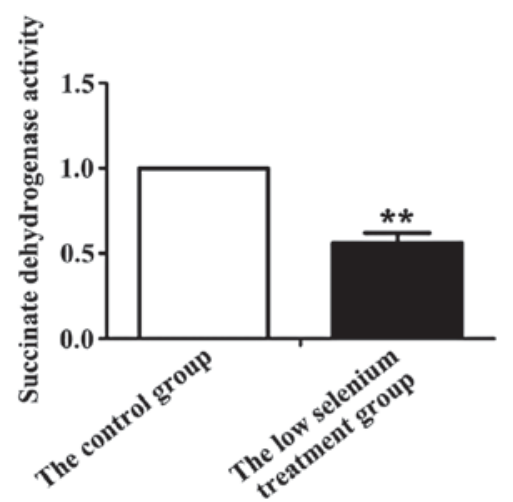

B

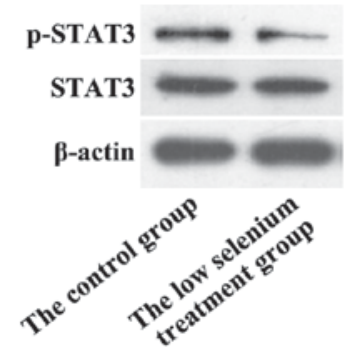

C

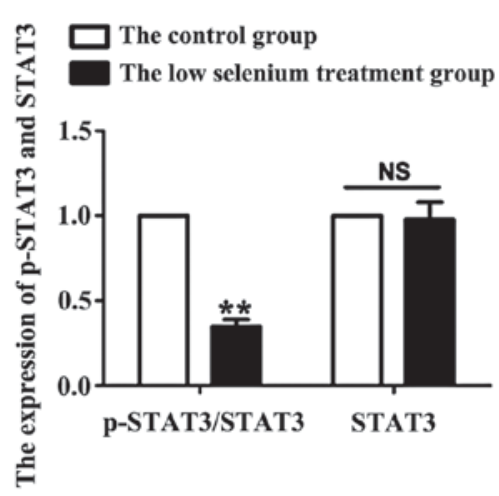

Figure. 1 Changes of succinate dehydrogenase activity and STAT3 expression. (A) Mitochondria from the control and low selenium treatment groups were extracted, and succinate dehydrogenase activity was measured. Succinate dehydrogenase activity in the low selenium treatment group was significantly decreased. (B) The levels of p-STAT3 and STAT3 in the control and low selenium treatment groups were measured by immunoblotting. (C) Statistical analyses showed that mitochondrial STAT3 activity (p-STAT3/STAT3) in the low selenium-treated group was significantly lower than that of the normal control group, but no significant change in STAT3 level was detected. ${ }^{* *} \mathrm{P}<0.01$. NS, no significant difference. STAT3, signal transduction and activator of transcription 3.
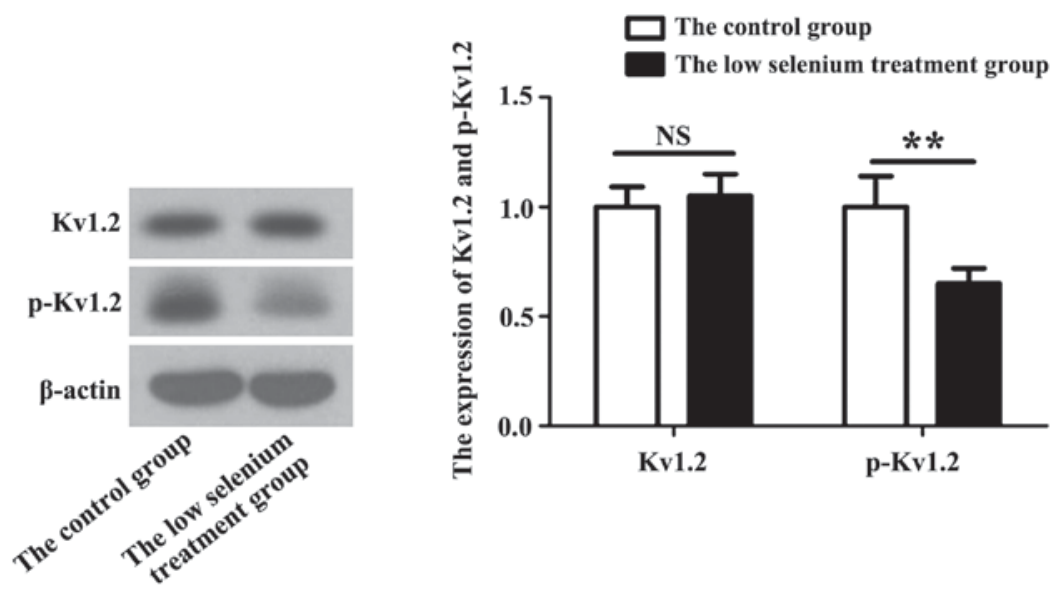

Figure 2. Expression of the potassium channel protein, $\mathrm{KCN}$, in the normal control and low selenium treatment groups. Expression of the Kv1.2 potassium channel and p-Kv1.2 in cardiomyocytes of the low selenium-treated group was measured by immunoblotting. Active Kv1.2 (p-Kv1.2) level in the low selenium-treated group was significantly lower than that in the normal control group. ${ }^{* * *} \mathrm{P}<0.01$.

expression of p-STAT3 and total STAT3 was assessed by immunoblotting. STAT3 activity is represented by the ratio of p-STAT3 (active state) to total STAT3. Mitochondrial STAT3 activity (p-STAT3/STAT3) in the low selenium treatment group was significantly lower than that in the normal control group $(\mathrm{P}<0.01)$, but there was no significant difference in STAT3 level between the two groups (Fig. 1B).

Potassium channel protein expression. Potassium channel expression in cardiomyocytes is diverse with different degrees of activity. We selected the Kv1.2 potassium channel as the detection index. Kv1.2 protein becomes active through an autophosphorylation reaction. The expression of Kv1.2 and p-Kv1.2 in myocardial cells of the low selenium treatment group was assessed by immunoblotting. Compared with the normal control group, the level of p-Kv1.2 in the low selenium-treated group was significantly lower (Fig. 2).

Detection of changes in apoptosis-related protein expression. $\mathrm{Bax}$ and $\mathrm{Bcl}-2$ are important factors in apoptosis. When apoptosis occurs, related signaling pathways become activated. Apoptosis can be determined by the expression pattern of these two proteins. Whole cell lysates from normal and low selenium treated myocardial cells were prepared, and the levels of Bax and Bcl-2 in primary cardiomyocytes were assessed by immunoblotting. As shown in Fig. 3, Bax protein level in the low selenium-treated group was significantly increased, while Bcl-2 protein level was decreased.

Flow cytometric detection of myocardial cell apoptosis in the low selenium-treated group. Cardiomyocytes were harvested from the normal control and low selenium-treated groups after $36 \mathrm{~h}$ of culture, and the apoptotic rate was determined by flow cytometry. PI staining showed that cells in the normal control group were mainly non-apoptotic, while the low selenium-treated group showed severe apoptosis and a large number of dead cells. After statistical analyses, we found that the apoptotic rate of cardiomyocytes in the low selenium-treated group was $42.62 \pm 2.21 \%$, which was significantly higher than that of the normal control group $(6.13 \pm 2.32 \%)$, indicating that 

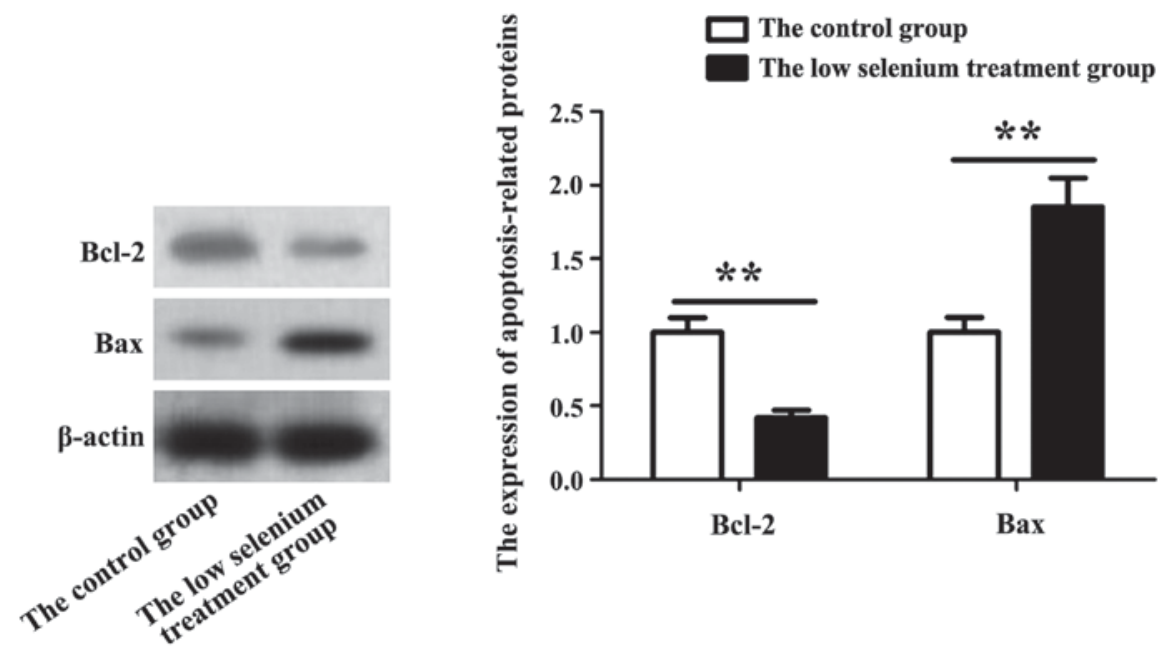

Figure 3. Changes in the expression of apoptosis-related proteins, Bax and Bcl-2. Expression of Bax and Bcl-2 in primary cardiomyocytes was assessed by immunoblotting. Expression of Bax was significantly increased, while the expression of Bcl-2 was decreased in the low selenium-treated group. ${ }^{* *} \mathrm{P}<0.01$.
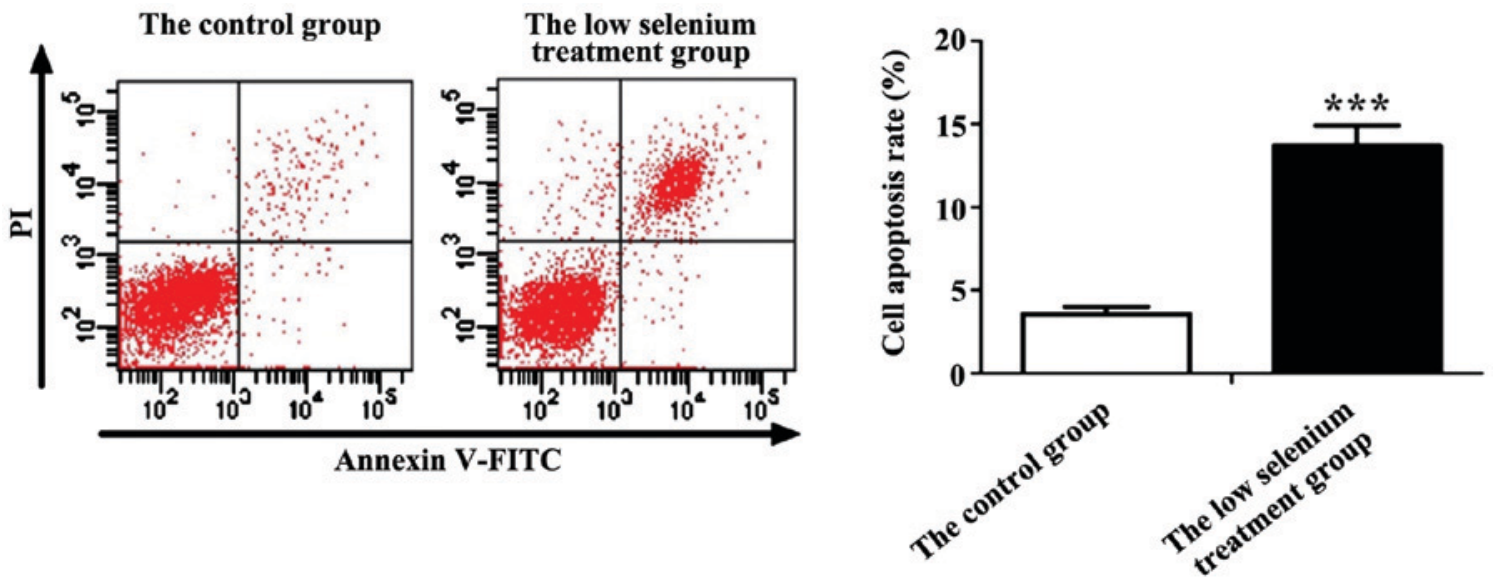

Figure 4. Apoptotic rate of the control and low selenium treatment groups detected by flow cytometry. ${ }^{* * *} \mathrm{P}<0.001$.

low selenium conditions have significant proapoptotic effects on cardiomyocytes (Fig. 4).

\section{Discussion}

As one of the essential trace elements of humans, selenium is related to various biological processes such as antioxidation, immunity, aging and disease (8). Selenium deficiency can cause a variety of diseases, most of which are caused by oxidative damage. Studies found that mitochondrial injury was the primary result of cardiomyocyte injury caused by decreased blood selenium level and is reflected by the damage of the mitochondrial membrane, generation of reactive oxygen species, and elevation of lipid oxide, eventually leading to apoptosis of cardiomyocytes (9). As a member of the mitochondrial signal transduction and transcription factor family, p-STAT3 is closely related to cell damage (10). Both clinical and experimental data have shown that the levels of STAT3 protein are reduced in cardiomyopathy patients and animal models, indicating the involvement of STAT3 in the pathogenesis of cardiomyopathy (11). A previous study found that STAT3 plays an important role in preserving electron transport chain complexes, and stabilizing intracellular ATP levels to maintain the normal function of mitochondrial protection through interaction with mitochondrial respiratory chain complex I (12). In addition, selenium can upregulate the activity of STAT3 in cells, which in turn regulates the expression of downstream genes and participates in the apoptotic process (12). Myocardial mitochondrial STAT3 activity was shown to be positively correlated with cardiac function in rats (13). In this study, we isolated cardiomyocytes from C57BL/6 neonatal mice. Cardiomyocytes were subjected to low selenium treatment to simulate the pathological environment of selenium deficiency. After mitochondria were isolated from cardiomyocytes, changes in p-STAT3/STAT3 were assessed by immunobloting to demonstrate changes in mitochondrial STAT3 activity. The results showed that STAT3 activity was decreased under low selenium conditions, although the expression of STAT3 protein did not change. Detection of succinate dehydrogenase activity can indirectly indicate changes of mitochondrial activity. Our results showed that mitochondrial activity in cardiomyocytes was significantly lower in the low selenium treatment group than in the control group. These data suggest that low selenium 
treatment may affect the activity of mitochondrial STAT3, leading to impaired mitochondrial function.

As the most diverse and widespread ion channels of cellular membranes, potassium channels are closely related to cytokine secretion, gene expression, proliferation, and apoptosis (14). The molecular structure of potassium channels and their function in anti-heart failure have been previously reported. However, the role of potassium channels in cell proliferation, division, and apoptosis remains unclear. The roles and functional mechanisms of potassium channels in cardiomyocyte injury remain unknown $(15,16)$. It was found that apoptotic rate was increased in cardiomyocytes with increased expression of cysteinyl aspartate specific proteinases (caspases), and potassium channels decreased the rate of apoptosis by reducing potassium influx (17). Studies have also reported that potassium channel blockers can inhibit cell apoptosis (18). These studies suggest that intracellular potassium efflux and apoptosis are closely related (19). There are various potassium channels with diverse functions (20). The role of the Kv1.2 potassium channel in myocardial disease remains unclear. STAT3 plays a protective role in the myocardial ischemia model. Potassium channels also play an important role in maintaining normal physiological function and inhibiting autophagy of cardiomyocytes. However, there is no report on whether the effects of selenium deficiency on the activity of mitochondrial STAT3 and Kv1.2 in cardiomyocytes can induce the generation of apoptotic signals, and further lead to cardiomyocyte apoptosis. Therefore, we isolated cardiomyocytes and treated them with low selenium to investigate this phenomenon at the cellular level. Kv1.2 level did not change significantly after low selenium treatment, although p-Kv1.2 level decreased significantly, indicating that the activity of potassium channels in myocardial cells decreased under low selenium conditions. The expression of the apoptosis-related proteins, Bax and Bcl-2, was measured under the same conditions where both STAT3 and potassium channels were affected. The expression of apoptosis-related proteins Bax was significantly increased while Bcl-2 was significantly decreased in the low selenium treatment group. Flow cytometry showed that the rate of apoptosis of cardiomyocytes was increased after treatment with low selenium.

In conclusion, selenium deficiency reduced mitochondrial STAT3 activity and reduced the activity of mitochondria. In addition, selenium deficiency reduced the expression of potassium channels in cardiomyocytes and promoted the expression of apoptosis-related proteins such as Bax, leading to the apoptosis of cardiomyocytes. This study further elucidated the pathogenic mechanism of selenium deficiency in myocardial diseases and provided a theoretical basis for the development of selenium-related therapeutic drugs.

\section{References}

1. Takeda T: Post-translational activation of non-selenium glutathione peroxidase of Chlamydomonas reinhardtii by specific incorporation of selenium. Bio Bio Rep 4: 39-43, 2015.

2. Vinceti M, Mandrioli J, Borella P, Michalke B, Tsatsakis A and Finkelstein Y: Selenium neurotoxicity in humans: bridging laboratory and epidemiologic studies. Toxicol Lett 230: 295-303, 2014.
3. Krohn RM, Lemaire M, Negro Silva LF, Lemarié C, Bolt A, Mann KK and Smits JE: High-selenium lentil diet protects against arsenic-induced atherosclerosis in a mouse model. J Nutr Biochem 27: 9-15, 2016.

4. Mchuron EA, Harvey JT, Castellini JM, Stricker CA and O'Hara TM: Selenium and mercury concentrations in harbor seals (Phoca vitulina) from central California: health implications in an urbanized estuary. Mar Pollut Bull 83: 48-57, 2014.

5. Guo X, Qiu J, Tu T, Yang X, Deng L, Anders RA, Zhou L and Fu YX: Induction of innate lymphoid cell-derived interleukin-22 by the transcription factor STAT3 mediates protection against intestinal infection. Immunity 40: 25-39, 2014.

6. Li H, Yang T, Long Z and Cheng J: Effect of mitochondrial ATP-sensitive potassium channel opening on the translocation of protein kinase $C$ epsilon in adult rat ventricular myocytes. Genet Mol Res 13: 4516-4522, 2014.

7. Molina ML, Giudici AM, Poveda JA, Fernández-Ballester G, Montoya E, Renart ML, Fernández AM, Encinar JA, Riquelme G and Morales A: Competing lipid-protein and protein-protein interactions determine clustering and gating patterns in the potassium channel from Streptomyces lividans (KcsA). J Biol Chem 290: 25745-25755, 2015.

8. Koc S, Cayli S, Aksakal C, Ocakli S, Soyalic H, Somuk BT and Yüce S: Protective effects of melatonin and selenium against apoptosis of olfactory sensory neurons: A rat model study. Am J Rhinol Allergy 30: 62-66, 2016.

9. Estevez AO, Morgan KL, Szewczyk NJ, Gems D and Estevez M: The neurodegenerative effects of selenium are inhibited by FOXO and PINK1/PTEN regulation of insulin/insulin-like growth factor signaling in Caenorhabditis elegans. Neurotoxicology 41 : 28-43, 2014.

10. Siveen KS, Sikka S, Surana R, Dai X, Zhang J, Kumar AP, Tan BK, Sethi G and Bishayee A: Targeting the STAT3 signaling pathway in cancer: Role of synthetic and natural inhibitors. Biochim Biophys Acta 1845: 136-154, 2014.

11. Atkinson GP, Nozell SE and Benveniste EN: NF- $\kappa$ B and STAT3 signaling in glioma: Targets for future therapies. Expert Rev Neurother 10: 575-586, 2014.

12. Lee JH, Chiang SY, Nam D, Chung WS, Lee J, Na YS, Sethi G and Ahn KS: Capillarisin inhibits constitutive and inducible STAT3 activation through induction of SHP-1 and SHP-2 tyrosine phosphatases. Cancer Lett 345: 140-148, 2014.

13. Wolfson RK, Mapes B and Garcia JG: Excessive mechanical stress increases HMGB1 expression in human lung microvascular endothelial cells via STAT3. Microvasc Res 92: 50-55, 2014.

14. Speca DJ, Ogata G, Mandikian D, Bishop HI, Wiler SW, Eum K, Wenzel HJ, Doisy ET, Matt L, Campi KL, et al: Deletion of the Kv2.1 delayed rectifier potassium channel leads to neuronal and behavioral hyperexcitability. Genes Brain Behav 13: 394-408, 2014.

15. Rahbek M, Nazemi S, Odum L, Gupta S, Poulsen SS, Hay-Schmidt A and Klaerke DA: Expression of the small conductance $\mathrm{Ca}^{2+}$-activated potassium channel subtype 3 (SK3) in rat uterus after stimulation with $17 \beta$-estradiol. PloS One 9: e87652, 2014.

16. Schwingshackl A, Teng B, Makena P, Ghosh M, Sinclair SE, Luellen C, Balasz L, Rovnaghi C, Bryan RM, Lloyd EE, et al: Deficiency of the two-pore-domain potassium channel TREK-1 promotes hyperoxia-induced lung injury. Crit Care Med 42: 692-701, 2014.

17. Kim GE, Kronengold J, Barcia G, Quraishi IH, Martin HC, Blair E, Taylor JC, Dulac O, Colleaux L, Nabbout R, Kaczmarek LK: Human slack potassium channel mutations increase positive cooperativity between individual channels. Cell Rep 9: 1661-1672, 2014.

18. Carrisoza-Gaytan R, Salvador C, Diaz-Bello B and Escobar LI: Differential expression of the Kv1 voltage-gated potassium channel family in the rat nephron. J Mol Histol 45: 583-597, 2014.

19. D'Adamo MC, Catacuzzeno L, Di Giovanni G, Franciolini F and Pessia $\mathrm{M}: \mathrm{K}^{+}$channelepsy: Progress in the neurobiology of potassium channels and epilepsy. Front Cell Neurosci 7: 134, 2013.

20. Dondapati SK, Kreir M, Quast RB, Wüstenhagen DA, Brüggemann A, Fertig N and Kubick S: Membrane assembly of the functional KcsA potassium channel in a vesicle-based eukaryotic cell-free translation system. Biosens Bioelectron 59: 174-183, 2014. 